\section{MEDICAL IONISATION: ITS USES AND POSSIBILITIES. ${ }^{1}$}

BY N. S. FINZI, M.B. LoND.,

MEDICAL OFFICER TO THE ELECTRICAI DEPARTMENT OF THE METROPOUTTAN HOSPITAL; MEDICAI OFFICER TO THE X RAY DEPAR' MENT OF THE GERMAN HOSPITAL; RADIOGRAPHER TO THE HOSPITAL FOR EPILEPSY AND PARAIYSIS, MAIDA VALE, W.

WHEN the secretary of the Asculapian Society wrote to me and suggested that the members of the society would care to hear something about ionisation, I readily acceded to his request for several reasons. In the first place, I have in certain diseases employed this method of treatment extensively in my work at the Metropolitan Hospital ; in the next, I am engaged in researches which I hope will some day open up new lines for the use of these very valuable remedies; and finally, if the principles are understood the treatment can be employed by any medical man with the assistance of very little apparatus and at comparatively small expense. I must here, however, emphasise the need of great care in the applications and a thorough mastery of the principles and of the technique, and these must be as rigidly observed as if a surgical operation were being undertaken.

By medical ionisation, or, as it is called abroad (and this is a much better name) "iontophoresis," we mean the introduction of ions of different sorts into the tissues of the human body. As you all no doubt know from your knowledge of chemistry, when metallic salts and certain other substances are dissolved in water a portion of the salt is split up into its component parts ; each of these parts carries an electrical charge, the acid radicle carrying a negative charge and the basic radicle a positive one. These minute particles are known as ions. It has been further found that the ions of any one substance always carry the same quantity of electricity, and the charge is the same for all bodies of the same chemical valency or combining power and varies directly as this valency. This is very important as we use it to calculate the amount of a substance introduced into the body. The splitting up of the salt is more complete the more dilute the solution, but up to a certain point there are more ions present in a strong solution as, though the proportion of ions to the complete salt is less, there is more of the salt present to be ionised. After a certain concentration the quantity of ions does not increase. If, now, in a vessel containing one of these solutions we place the wires from a battery or from some source of constant electrical current, all the positively charged ions (which we have seen are the basic radicles) will be repelled from the positive pole and move towards the negative pole at a rate dependent on the voltage of the current. Similarly, all the negatively charged ions (acid radicles) will move away from the negative pole. Now, if we imagine a patient completely separating our solution into two parts, with one pole of the battery in each, the negatively charged ions in their struggle to get away from the negative pole will rush into the patient to make for the positive pole, from which, in its turn, the positively charged ions will be getting into the other side of the patient, who may be considered as a porous bag of saline solutions containing many ions. When these foreign ions get into him they displace ions carrying electricity of the same sign in his own salts, these in turn displace others, and at his opposite side some of his own ions pass out into the solution. If the experiment were continued long enough a certain proportion of the introduced ions would pass out the opposite side with these; but that never happens in practice. There is no necessity for the solution to be the same on both poles; thus, we may at one time have potassium iodide on the negative pole and introduce iodine, and sodium chloride on the positive pole and introduce sodium. The pads containing the solutions must not touch one another at any point as the current would then pass from solution to solution without any appreciable quantity going through the patient.

Having given a general idea of what ionisation is, I now pass on to the properties of ions. I want it to be realised in the first place that ions have not the properties of the same substance without an electrical charge. This substance

1 A paper read before the Asculapian Society on Jan. 8th, 1909. ordinarily consists of uncharged atoms or molecules. The ions bear more resemblance in their properties to solutions of the compounds which contain them in the combined condition. Thus, chlorine ions have no resemblance to chlorine in its ordinary state but resemble it as it exists in chloride of sodium solution, in which, of course, it is chiefly in the ionic state in combination with sodium ions.

The next thing to remember is, that as soon as the ions get into the tissues they enter into combination with the ions they find there, and in some cases they lose their charge for instance, if a precipitate is formed. The effect of thi may be that they offer a resistance to the passage of further ions. I show a section of the skin of a cat into which I have attempted to pass ferric ions. The skin was placed in potassium ferrocyanide solution to stain the ferric ions in situ and was then hardened in alcohol. It will be seen that, although the current was passing for a considerable time, the ions have partly penetrated the epidermis and corium but have not reached the connective tissue. In this experiment it was always found that the voltage had to be increased continually after the first few seconds to keep the current constant, showing that the resistance must have also increased. Zinc ions are also precipitated, but in the corium and subcutaneous tissues, and probably in the form of phosphates. The same thing occurs with calcium. I have placed under the microscope a piece of skin ionised with calcium. You will see the granules of precipitate, stained deeply, infiltrating the corium and subcutaneous tissue. Another cause of precipitation is the decomposition of the ion itself. This happens when permanganate ions are driven in, as they are immediately decomposed into manganese dioxide and oxygen, the latter going to the tissues. Other ions-e.g., potassium, sodium, lithium, ammonium, chlorine, iodine, sulphate, ferrocyanide \&c., pass in the soluble state into the tissues. There is section showing the last under the microscope, the ferrocyanide having been stained by soaking the tissue in a ferric solution. You will see that these ions pass through even the subcutaneous capillary network.

A simple experiment to show the difference between ion and their compounds can be performed by anyone who has a galvanic battery. The properties of cocaine hydrochloride, which do not differ pharmacologically from those of cocaine itself, are well known. But if a person ionises cocaine into himself (cocaine is a base and therefore goes in at the positive pole) a difference is noticed in a short time; the skin becomes anæsthetic, but the smarting sensation caused by the galvanic current is felt just the same as before. So far the effects seem the same, but if the ionisation is now stopped it will be found in a short time that the ionised patch becomes very tender, hyperæsthetic, and hyperæmic, and remains so for some while. If sufficient cocaine has been driven in this hyperæmia persists and is replaced later by a brown pigmentation. I do not know whether eucaine and other local anæsthetics act in the same way, but so far as cocaine is concerned ionisation is useless for local anæsthesia on account of the short duration of anæsthesia and the subsequent hyperæsthesia.

Ions may be used for their general effects, or for their local effects, or for both. The general effects are pretty much the same as in oral administration of solutions contain ing the same ions, but ionised drugs act more slowly and continue acting much longer owing to their being only gradually disengaged from the combinations which they form in the tissues into which they are driven. Thus, Brouillet, ${ }^{2}$ in one experiment, during which he drove into himself 0.1854 gramme (about 23 grains) of iodine, found the drug in the saliva 14 minutes from the commencement of the application, while it did not disappear for 75 hours and was detected in the urine for 68 hours. On a subsequent occasion, when he drove in 0.2162 gramme ( 34 grains), excretion was continued for 49 hours in the saliva and 33 hours in the urine.

For purely general effects oral administration of the drug, ionised as much as possible by being dissolved in a large quantity of water, will, on account of its simplicity, be generally used. Many ions used for local medication, how ever, will pass into the circulation and act also on the system generally. The local uses are the most important and fall Fils. 
into two classes-the action on the skin and that on the deep parts, such as joints, muscles, tendons, \&c. Another local use is to extract ions from the tissues-for instance, uric acid ions in gout.

In using a fresh ion we require to know whether it passes through the epidermis, and, if it does, whether it is deposited in the corium or driven into the deeper tissues. This can only be definitely determined by experiments on animals with skin as like our own as possible. It is necessary to obtain the ion in a solution which will not damage the part treated, and this is generally done by having a dilute solution. In some cases, however, we cannot obtain such a solution. I want to use bismuth ions in the treatment of ulcers : a double salt (e.g., bismuth ammonium citrate) will not do as the tissues offer more resistance to the passage of ions of heavy metals than the alkalies, and bismuth would probably not be driven in to any extent, the ammonium going in instead. The other bismuth salts are only soluble in excess af acid; the acid must be so strong that its application to the ulcer would be too painful for practical use and so $I$ have not been able to use bismuth ions in these cases at present. If an ion is deposited in the epidermis or corium its effect must, of course, be local, at any rate chiefly, and this is the case with most of the heary metals. Most acid radicles, however, pass easily through the epidermis into the deep tissues, and the ions of the alkaline metals do the same. Many organic salts also form ions, having their own special properties.

A careful technique is most important. All greasy applications, being non-conductors of electricity, must be carefully removed or all the current will pass through the small patches of the surface which have escaped the grease and electrolysis of the skin will follow. The electrode must press firmly and evenly on the surface, and for this purpose consists of a great thickness of absorbent cotton saturated with the solution to be employed and a metallic electrode of as flexible a nature as possible over it. The copper chain mail electrode (supplied by Schall) is one of the most convenient, but should consist of a metal less easily attacked, such as tin: gold or platinum would be far better, but they are, of course, too expensive. The next best electrode is tinfoil, on account of its extreme flexibility, the difficulty with which it is chemically attacked and its comparative cheapness. Metallic gauzes are also very suitable. The pad with the metal over it is then bandaged firmly on to the part, the cotton being always wider than the metal. For uneven parts, such as the fingers or toes, extra thickness of cotton must be used.

It is advisable to wash one's own fingers after touching one electrode in order to avoid contaminating the other. If it is necessary to limit the action to a small area, this is done by placing in contact with the skin a piece of jaconet, oilsilk, or strapping with a window of the necessary size cut in it and the pad over it. Any abrasion on the skin must be avoided, and, if it is necessary to ionise over it, must be sealed with a drop of collodion, which is allowed to dry. The reason of this is that if the skin is broken its resistance is diminished and the current, choosing the easiest path, becomes very dense at the abrasion. Excessive density of current on any part of the skin will after a time damage it, and if the patient complains of feeling the smarting more at one spot, or the current cannot be raised as high as usual on account of pain, it is essential after a few minutes to take the electrode right off and examine for any specially hyperæmic place. The corner of a rather stiff electrode sometimes gets bent down, pressing more heavily on the cotton, and this may often be the cause. If any damage to the skin occurs the part is covered with dry sterile wool and in a few days an aseptic scab is formed, under which the skin soon heals.

The current is supplied from batteries or from a continuous current main and regulated by means of a shunt rheostat by which the voltage can be varied from 0 to 80 . It must be turned on and turned off very gradually, as it is the increase and decrease of the current that is painful. There always remains some smarting, but during a long sitting this almost disappears.

We are, it will be remembered, bound to introduce some substance at each pole, though they may be placed at widely distant parts of the patient's body. Plain water must not be used as the resistance is very great, and either the high voltage required or the hydrogen and hydroxyl ions are very irritating, and so when only the effect of one ion is required we introduce some innocuous substance, such as chlorine or ammonium, at the other pole. The amount of current that can usually be borne without serious discomfort is about one milliampère per centimetre of the periphery of the electrode or jaconet window as the case may be.

And now I come to the therapeutics of iontophoresis. With regard to doses, the amount driven in depends on the amount of current and the time, and is the same whether we use one milliampère for 10 minutes or 10 milliampères for one minute.

Weight driven in in grammes = electro-chemical equivalent $x$ current $x$ time

Electro-chemical equivalent $=\underline{\text { atomic weight } \times \cdot 000010384}$ valency

Stephane Leduc, who placed this method of treatment on a scientific basis by showing what actually occurred in "cataphoresis," mentions especially three different ions : (1) chlorine for causing absorption of fibrous tissues-sclerolysis; (2) zinc for the treatment of rodent ulcer, ulcer, abscess, endometritis, and other conditions ; and (3) salicylic ions for the treatment of neuralgias and rheumatism. He also gave us uses for several other substances. Dr. H. Lewis Jones did great service by introducing Leduc's work into this country. The value of chlorine ions for sclerolysis is not proved, but they are probably of use if combined with passive movement immediately after the application. The substance is introduced from large pads saturated with ammonium or sodium chloride solution. A sitting of an hour with from 50 to 80 milliampères is given three times a week or, better, half an hour every day. Chlorine ions have besides been used for corneal opacities. The other halogen used at present is iodine. This also has been used for sclerolysis with advantage, ${ }^{3}$ and for rheumatoid arthritis, gout, synovitis, \&c. In rheumatoid arthritis and gout $I$ use it at the same time as lithium on the other pole and will refer in detail to this presently. Iodine has further been used with success in syphilis. I have a case of tertiary ulcers under treatment in which $I$ have several times prevented new gummata from breaking down, and though there has been much improvement $I$ have not yet been able to cure the ulcers. In another tertiary ulcer which I have started treating only recently great improvement has already resulted. In a case of tabes dorsalis with intense pain (girdle and lightning) great relief was afforded. Fluorine and bromine ions are not used at present, but it is quite probable that they will have their uses when their pharmacology has been worked out. Salicylic ions are recommended for neuralgias and have been successfully employed, even in tic douloureux. I have one such case under my care at present in which they have given great relief after quinine ions had failed, but as the cause is probably not attacked[ am afraid that it will do no more than delay the excision of the Gasserian ganglion. Nevertheless, the result so far is good enough for one to hope that mild cases may be cured without surgical measures. Successes have also been claimed for salicylic ions in rheumatoid arthritis. I have seen improvement in some cases, but have discarded this substance for iodine and lithium. Among other negatively charged ions may be mentioned pyrogallic ions, with which Neumann and Volk ${ }^{4}$ claim to have cured cases of lupus, and in so modest and careful a paper that it must receive our serious consideration.

Leaving the acid radicles now, we come to the basic ones. Ammonium ions cause less discomfort than any other basic ions, but neither ammonium, potassium, nor sodium seems to have any therapeutic or pharmacological effect, and they are therefore used when no effect is required from the positive ions. Lithium on the positive side, in conjunction with iodine on the negative side, I have found most useful in gout and rheumatoid arthritis. I tried salicylic ions in the latter with only a little benefit. I next used iodine and ammonium ions and got rather better results. Finding that the radiographic changes in gout are all found in rhenmatoid arthritis, though the order of onset is generally different, I decided to try lithium. This gave about as much relief as iodine and then $I$ used the two at and 20 th, 1907 . 
the same time with splendid results, both in gout and rheumatoid arthritis. I have had two cases of acute gout to treat by this method and the relief was immediate. The agonising shoots of pain disappeared after the first application and the slight tenderness which remained soon vanished after a second. Needless to say, the cedema rapidly disappeared after the first. The immediate relief is one of the most marvellous things in medicine. A case of monarticular arthritis, probably rheumatoid, who had been treated by other methods for a couple of years with little success, and who could only walk with the aid of a stick when he came, was so far recovered after two and a half months as to be able to take a 30 -mile walk and return to his duty as a policeman.

How far can a rheumatoid arthritis or gout be cured? It is, of course, at present impossible to answer this question, but it does not seem likely that we can cause the absorption of bony deposits, and we certainly cannot get rid of bony ankylosis. With regard to the cavities in bone so commonly found, however, the case is different, and one hopes in time to get these filled up with normal bone. It is yet too early to say anything definite, especially as the patients get so much better that they return to work and cease attendance at the hospital.

Zinc has been more used for iontophoresis than any other ion. In rodent ulcer the zinc ions seem to have a selective action on the growth, causing the skin to heal over and the nodules to disappear. In America it has been used for large epitheliomas; a general anæsthetic is given and a very large current used. In a case of fungating epithelioma which was ionised with zinc I have found considerable fibrosis in the ionised parts. The growth was, however, too big to be dealt with even by heroic measures.

In rodent ulcer care must be taken to get rid of all the nodules. The healthy skin is protected by jaconet or oilsilk and a current of three milliampères per square centimetre passed for from 25 to 30 minutes. At the end of the application the ionised part will be a glistening white colour. A certain amount of inflammation follows which disappears in five or six days, the ulcer rapidly heals, and the nodules shrink away. If any nodules are left the application must be repeated. In the majority of cases I prefer massive doses of $x$ rays for this disease becanse the are painless; they search out nodules of the disease which cannot be seen or felt, and if a carefully measured full dose is given every 17 to 22 days there seems no danger of producing telangiectases as in the old method of frequent small doses. The scar in either case is very good. Employing much smaller doses of zinc I have had most excellent results in chronic ulcers of the leg, especially the varicose variety the only other treatment used is a dressing of vaseline and a properly applied calico bandage. The patient goes about his or her work as usual. I have been able to cure cases of many years' standing and cases treated by every other means. The most satisfactory thing is the immense relief to pain afforded after the first few hours, which persists for many days.

Zinc ions appear to be useful for all similar conditions such as erosion of the cervix, fissure of the anus, mucous colitis, and chronic sinuses. Copper ions have been used for sinuses with success, but I have not found them of much use in ulcers. They have been recommended for ringworm, and in the one case in which I used them $I$ obtained a cure, and there was no recurrence six months afterwards. Hartigan has also reported successes in lupus erythematosus with copper. Magnesium ions are very useful for the ordinary multiple warts that one sees on the hands, though on some varieties they appear to have no effect.

Such, then, are the uses to which iontophoresis has already been put and they are both numerous and varied. There is a great field for its use in other skin diseases and also in affections of joints and muscles. For instance, I should think that sulphur ions (from, say, sulphide of soda) would be useful in many chronic skin affections but have had no opportunity to try it. Further, it is the only non-vital method we have for extracting substances from the tissues, all the other methods aiming at stimulating the excretory giands. There has been of late years a growing prejudice against the use of drugs. Some people prefer to administer an extra dose of the same poison which is causing the symptoms, and others to fill the diseased part up with effete products by obstructing the circulation. The pendulum will, however, swing back and drugs will again become fashionable, but in many cases in this more efficient form, and the old local applications will be a thing of the past.

The uses of iontophoresis are certain to be very considerably extended in a few years, so much so that the battery will become as common as the stethoscope and every student will be taught its uses. At present, however, the specialists must still hold the field, and it is for us to work out the applications of the treatment and by establishing the pharmacology of ions to lift their use from the realms of empiricism and place them on a solid pedestal of fact from which they can never be dethroned.

Welbeck-street, W.

\section{A CASE OF PRIMARY OVARIAN ACTINOMYCOSIS. ${ }^{1}$}

BY FRANK E. TAYLOR, M.D. LOND., M.R.C.P. LOND., F.R.C.S. ENG., D.P.H. CAMB.,

GYNACOLOGIST TO THE HAMPSTEAD GENERAL AND NORTH-WHSI LONDON HOSPITALS; OBS'LETRIC REGISTRAR AND TUTOR, THE MIDDLASFX HOSPITAL; LATE PATHOLOGIST TO THE CIIELSEA HOSPITAL FOR WOMLA, IONDOX;

$$
\text { AND }
$$

WELBY E. FISHER, F.R.C.S. ENG.,

LATE RESIDENT MEDICAL OFFICER, CHELSEA HOSPITAL FOR

$$
\text { WOMEN, LOXDOX. }
$$

ACTINomycosis is one of the rarer forms of infection of the human tissues, whilst actinomycosis of the ovary constitutes one of the curiosities of gynæcology, only six cases of this condition being on record. In none of these has the ovary been the primary seat of infection but has been infected by extension of the disease originating either in the intestines or in the vagina, uterus, and Fallopian tubes. In the case now recorded the ovary was the primary seat of the disease : it is, therefore, a unique case.

A woman, aged 34 years, single, was admitted into Chelsea Hospital for Women, under the care of Dr. W. H. Fenton, on June 24th, 1908, complaining of abdominal pain. Menstruation commenced at the age of 11 years and was associated with severe pain in the lower abdomen but was quite regular until the age of 17 years, when the patient's health failed; she became thin and drowsy and menstruation was completely arrested for 18 months. Her health then improved, she became fatter and stronger, and menstruation returned but was now very irregular and very profuse, the periods lasting from 10 to 14 days and requiring two or three dozen diapers, the intervals varying from five days to three months. About October, 1904, the patient being then 30 years of age abdominal pain was first noticed. It was felt in the right hypogastrium, being very severe and throbbing in character, and was aggravated by the recumbent posture and relieved by walking. About this time she also began to sweat profusely at nights and this continued until admission. After suffering in this way for about six weeks the patient saw Dr. Fenton, who found the right ovary enlarged; he diagnosed an ovarian cyst and recommended admission into hospital with a view to operation. This was declined. The pain and discomfort continued intermittently for 12 months, the patient meanwhile continuing at work. She then began to suffer excruciating pain on micturition, and for two months passed blood and pus in great quantity in the urine, which also contained small fleshy masses of about the size of grapes. A London practitioner was then consulted. He informed the patient that she had a displaced womb and ordered medicine and hot douches and inserted a ring pessary which had to be removed in three days on account of the pain caused thereby. In a couple of months, the pus having then disappeared from the urine, the pessary was reinserted. It now gave relief and was worn for three months. There was no vaginal discharge. The temperature was never taken. The bowels as a rule were constipated. In February, 1908, the patient had a very severe flooding and a fainting attack, and the right side from the hip to the toes was disabled by cramp which was present continuously night and day for 14 days. The same medical man was again consulted and he reinserted the pessary. There was now a thick yellow vaginal dis. charge, and although the ring was removed in a few days

1 A paper read at a meeting of the Gynecological and Obstetrical Section of the Royal Society of Medicine on March 11th, 1909. 